(2) Open Access Full Text Article

\title{
High expression of Sox 10 correlates with tumor aggressiveness and poor prognosis in human nasopharyngeal carcinoma
}

This article was published in the following Dove Press journal:

OncoTargets and Therapy

21 March 2016

Number of times this article has been viewed

\section{Yu Zhao ${ }^{1, *}$ \\ Zhi-gang Liu',* \\ Jiao Tang' \\ Ren-fang Zou' \\ Xiao-yan Chen ${ }^{2}$ \\ Guan-min Jiang ${ }^{3}$ \\ Yan-fang Qiu' \\ Hui Wang'}

'Key Laboratory of Translational Radiation Oncology, Department of Radiation Oncology, ${ }^{2}$ Department of Pathology, ${ }^{3}$ Department of Clinical Laboratory, Hunan Cancer Hospital, The Affiliated Cancer Hospital of Xiangya School of Medicine, Central South University, Changsha, Hunan, People's Republic of China

*These authors contributed equally to this work

\footnotetext{
Correspondence: Zhi-gang Liu; Hui Wang Key Laboratory of Translational Radiation Oncology, Department of Radiation Oncology, Hunan Cancer Hospital, The Affiliated Cancer Hospital of Xiangya School of Medicine, Central South University, Changsha 4210013, Hunan Province, People's Republic of China Email zhigangliu1983@hotmail.com; wanghui710327@163.com
}

Purpose: The aim of the study was to detect the expression of Sox10 in human nasopharyngeal carcinoma (NPC) and investigate the relationship between its expression and the clinicopathological characteristics of NPC patients.

Patients and methods: Tumor specimens $(n=105)$ were retrospectively collected from patients with NPC diagnosed between 2004 and 2005 who presented at Hunan Cancer Hospital. Immunohistochemistry analyses were performed to characterize the expression of Sox10 in NPC. Kaplan-Meier survival and Cox regression analyses were employed to evaluate the prognosis of 105 NPC patients.

Results: The results showed that Sox10 was markedly overexpressed in human NPC tissues. Analysis of clinicopathological parameters showed that high Sox10 expression was significantly correlated with the clinical stage $(P=0.032)$, T classification $(P=0.034)$, and lymph node metastasis $(P=0.03)$. Cox regression analyses further showed that Sox10 expression was an independent prognostic factor for overall survival $(P=0.005)$. This is the first time Sox 10 has shown its importance in predicting NPC progressiveness and survival outcomes.

Conclusion: Sox 10 serves as a potential biomarker for NPC patients. It may hopefully become a novel therapeutic target for NPC patients.

Keywords: Sox10, NPC, prognosis

\section{Introduction}

Nasopharyngeal carcinoma (NPC) is a highly invasive malignancy that rises from the epithelial lining of the nasopharynx. With an estimated 86,700 new cases and 50,800 deaths in 2012, NPC is viewed as a relatively rarer form of cancer globally. However, in endemic regions, including Southern China and Southeast Asia, the annual incidence rate reached $15-50 / 100,000 .{ }^{1}$ Although early stage NPC can be managed with high cure rate under radiotherapy, $70 \%-90 \%$ of NPC patients, at the time of initial diagnosis, are characterized by cervical nodal metastasis., ${ }^{2,3}$ The prognosis of NPC has improved a lot with the use of radiotherapy and chemotherapy, but for locally advanced and metastatic NPC, the result remains unsatisfactory due to local relapse and distant metastasis. ${ }^{4}$ An urgent need exists to identify novel and specific biomarkers that have clinicopathologic and prognostic values in NPC, and hopefully, help to guide the development of new therapeutic regimens.

Transcription factors of the Sox (sex-determining region Y-box protein) protein family, characterized by a conserved high mobility group DNA binding domain, regulate many diverse developmental processes as well as control homeostasis in adult tissues. ${ }^{5}$ There were 20 Sox genes identified. Among these, Sox 10 gene was the first Sox 
gene discovered to be involved in neural crest development. It functions by directing the activity of other genes that signal differentiation of neural crest into other cells, so it is essential for the survival of neural crest precursor cells and for proper differentiation of neural crest-derived melanocytes and glia. ${ }^{6}$ The association between Sox 10 mutation and myelination deficiency in human being's Waardenburg-Hirschsprung syndrome and disruptions of neural crest development has been established. ${ }^{7}$

In the last few years, an increasing amount of evidence has revealed that dysfunction and mutations of Sox factors are implicated in several human diseases, including a variety of cancers. These cancer cells are mostly originated in tissues overlapping with their expression pattern during embryonic development. The involvement of Sox10 in human cancer has been almost exclusively in melanomas and some brain tumors. ${ }^{8}$ Sox 10 activates the expression of microphthalmiaassociated transcription factor, a well-established oncogene in melanoma, and high expression of Sox10 in melanoma has been found to be involved in metastatic potential of melanoma. ${ }^{9-11}$ However, currently, little information has been addressed concerning the association between Sox 10 and carcinogenesis and progression of NPC.

In the present study, for the first time, we investigated Sox10 expression in NPC tissue specimens by immunohistochemistry (IHC) analyses. Besides this, through further analyses, we studied the relationship between Sox 10 expression and NPC clinical parameters, as well as the value of Sox10 in predicting NPC prognosis.

\section{Patients and methods Study patients and treatments}

Between May 2004 and July 2005, 105 consecutive patients with undifferentiated nonkeratinizing nasopharyngeal carcinoma NPC in the Department of Radiotherapy, the Affiliated Cancer Hospital of Xiangya School of Medicine, Central South University, Changsha, People's Republic of China, were enrolled retrospectively in this study. The medical ethics committee of Hunan Cancer Hospital approved the present retrieval method of cancer specimens. The criteria are listed as follows: histologically confirmed NPC, age ranging from 17 years to 77 years, managed in accordance with a uniform protocol, enough available tissue, and clinical follow-up. All patients underwent restaging according to the 2007 International Union against Cancer tumor-node-metastasis (TNM) classification. All patients received conventional two-dimensional radiotherapy. Radiotherapy was administered five times a week at a dose of 2 Gy/day. The accumulated dose of radiation ranged between 66 Gy and 78 Gy. In all, 34 patients were treated with boost dose to skull base. Patients did not receive chemotherapy unless metastasis or recurrence occurred. The primary end point was overall survival. Overall survival was defined as the time (in months) from the date of admission to the date of death from any causes or the last follow-up. The follow-up period ended on December 31, 2010, with a median follow-up of 61 months (range 9-80 months).

\section{$\mathrm{IHC}$ and evaluation of immunohistochemical staining}

Detailed steps for IHC were following the manufacturer's protocol. Briefly, antigen retrieval was performed in citrate buffer ( $\mathrm{pH}$ 6.0) for 20 minutes using a microwave oven. After blocking in 10\% normal blocking serum at room temperature for 10 minutes, slides were incubated with Sox10 antibody (1:200; Santa Cruz Biotechnology Inc., Dallas, TX, USA) at $4^{\circ} \mathrm{C}$ overnight. Negative (omission of the primary antibody) and positive controls were included according to manufacturer's datasheet of each antibody.

Semiquantitative assessment of the staining, including analysis of both the intensity and percentage of the stained cells, was used. The staining intensity was scored as $0,1+$, $2+$, or $3+$ corresponding to colorless, buff, brownish yellow, and dark brown, respectively. The percentage of positive stained cells at each intensity was estimated. Final scores ranging from 0 to 300 was calculated by multiplying the intensity and the percentage scores. The cutoff value for Sox 10 was chosen based on a measure of heterogeneity using the log-rank test statistical analysis with respect to overall survival. An optimal cutoff value was identified: a staining index of $\geq 14$ was used to define tumors of high expression and $\leq 13$, tumors of low expression.

\section{Statistical analysis}

SPSS 18.0 software (SPSS Inc., Chicago, IL, USA) was used for statistical analysis. The Pearson's chi-squared test was used to determine the correlation between Sox 10 expression and clinical factors. Survival curves were plotted using the Kaplan-Meier method and compared with the log-rank test. Statistically significant $P$-value was set as $<0.05$.

\section{Results \\ Sox 10 is highly expressed in NPC patients}

To determine Sox10 expression in clinical samples, we performed IHC analysis in 105 NPC specimens and adjacent tissues. It was revealed that Sox 10 was expressed in $84.8 \%$ 
of tumor samples ( 89 of 105). In contrast, the adjacent tissues exhibited undetectable or low Sox10 staining (Figure 1).

\section{Sox 10 is associated with clinical aggressiveness in NPC patients}

To elaborate the relationship between Sox10 expression levels and clinicopathological characteristics, 105 paraffinembedded NPC tissue specimens were selected for Sox 10 IHC staining. A summary of Sox 10 expression and clinicopathological parameters is presented in Table 1 . No statistically significant associations were observed between Sox 10 expression and sex, age, or N classification at the initial diagnosis $(P>0.05)$. However, high Sox 10 expression was significantly correlated with the clinical stage $(P=0.032)$, $\mathrm{T}$ classification $(P=0.034)$, and advanced $\mathrm{N}$ stage $(P=0.03)$.

\section{Sox 10 expression is closely correlated with adverse NPC overall survival time}

To evaluate the prognostic importance of high Sox10 expression in patients with NPC, we used Cox proportional hazards regression model and Kaplan-Meier method. According to
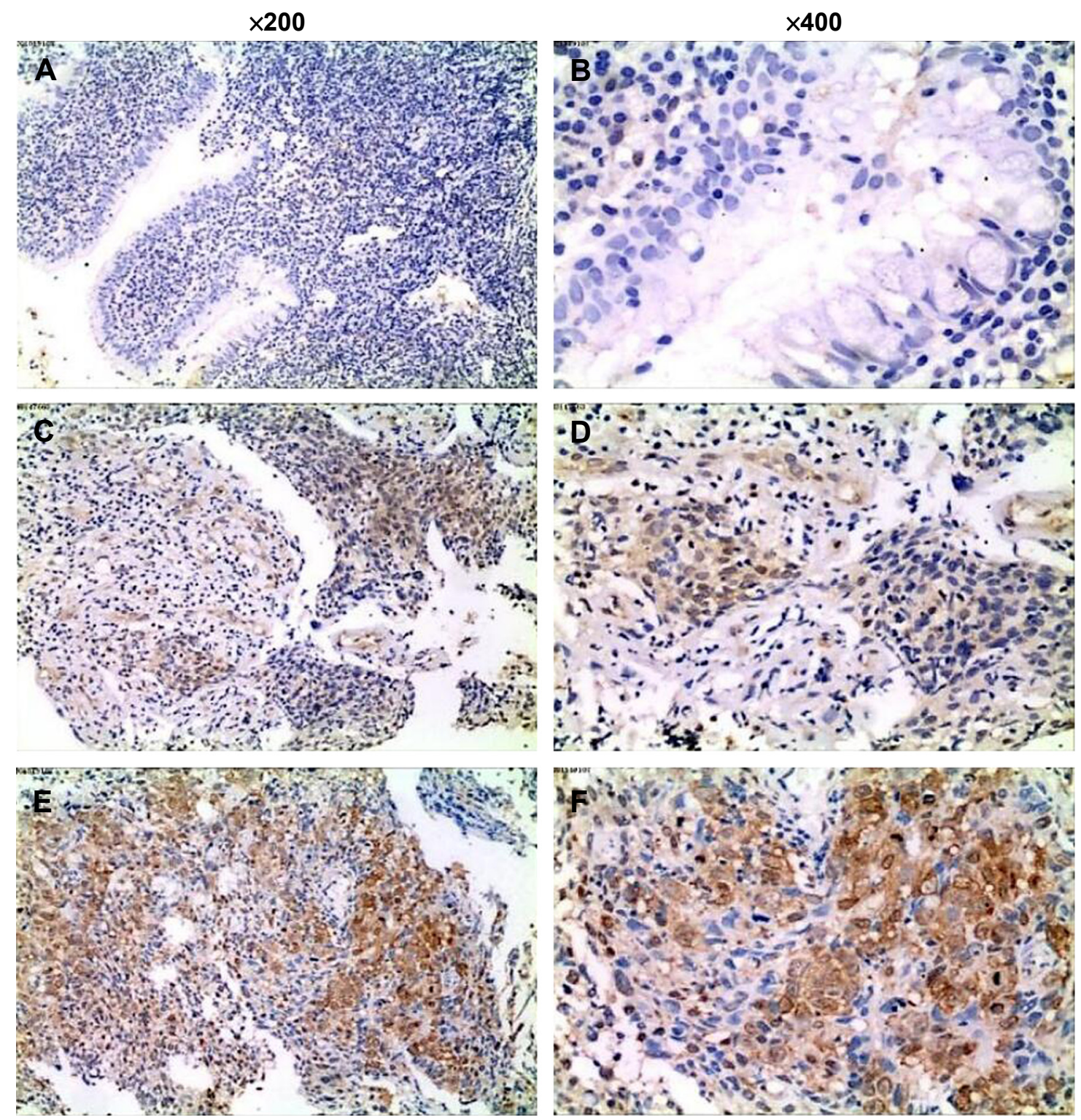

Figure I Sox 10 expressed in NPC tissue and adjacent nasopharyngeal tissue.

Notes: (A and B) Only a small amount of Sox 10 expression in adjacent nasopharyngeal epithelial cells (final score 4 ; magnification $\times 200$ and $\times 400$, respectively; hematoxylin). (C and D) Low Sox 10 expression in nasopharyngeal carcinoma tissue (final score II; magnification $\times 200$ and $\times 400$, respectively; hematoxylin). (E and F) High SoxI0 expression in nasopharyngeal carcinoma tissue (final score 240 ; magnification $\times 200$ and $\times 400$, respectively; hematoxylin).

Abbreviation: NPC, nasopharyngeal carcinoma. 
Table I Correlation between Soxl0 expression and clinicopathologic characteristics of NPC

\begin{tabular}{|c|c|c|c|}
\hline \multirow[t]{2}{*}{ Characteristics } & \multicolumn{2}{|c|}{ Sox 10 expression } & \multirow{2}{*}{$\frac{\text { Chi-square test }}{P \text {-value }}$} \\
\hline & $\begin{array}{l}\text { Low } \\
\text { expression }\end{array}$ & $\begin{array}{l}\text { High } \\
\text { expression }\end{array}$ & \\
\hline Age (years) & & & 0.277 \\
\hline$\leq 50$ & 40 & 37 & \\
\hline$>50$ & 11 & 17 & \\
\hline Sex & & & 0.404 \\
\hline Male & 32 & 39 & \\
\hline Female & 19 & 15 & \\
\hline Clinical stage & & & 0.032 \\
\hline I & 3 & 0 & \\
\hline II & 19 & 12 & \\
\hline III & 19 & 32 & \\
\hline IV & 10 & 10 & \\
\hline T classification & & & 0.034 \\
\hline $\mathrm{TI}$ & 9 & 2 & \\
\hline T2 & 28 & 30 & \\
\hline T3 & 5 & 13 & \\
\hline $\mathrm{T} 4$ & 9 & 9 & \\
\hline $\mathrm{N}$ classification & & & 0.138 \\
\hline No & 10 & 7 & \\
\hline $\mathrm{NI}$ & 25 & 18 & \\
\hline N2 & 15 & 28 & \\
\hline N3 & 1 & $\mathrm{I}$ & \\
\hline N0-I vs N2-3 & & & 0.03 \\
\hline NO-I & 35 & 25 & \\
\hline N2-3 & 16 & 29 & \\
\hline
\end{tabular}

Abbreviation: NPC, nasopharyngeal carcinoma.

univariate analysis, the overall survival of the NPC patients was correlated with Sox10 expression $(P=0.005)$ and age $(P=0.027)$. Multivariate analysis showed that Sox 10 may be viewed as an independent predictor for NPC overall survival $(P=0.011$; Table 2). Kaplan-Meier method and the log-rank test also showed a significantly inverse correlation between high Sox10 expression level and patient overall survival $(P=0.004)$, revealing that higher levels of Sox 10 expression were associated with shorter survival rate (Figure 2). These results, all together, revealed that Sox 10 may be a potential biomarker for the prediction of prognosis of NPC patients receiving radiotherapy.

\section{Discussion}

Characterized by a conserved high mobility group DNA binding domain, the Sox family is a group of related transcription factors that have demonstrated their importance in chondrogenesis, hematopoiesis, neural crest development, and neurogenesis. Over the years, continuous studies have revealed that many genes first identified as key genes in cancer development play crucial roles in embryogenesis. Conversely, many of the proteins controlling embryonic development are also involved in carcinogenesis, indicating that both processes are closely related. ${ }^{12}$ An increasing number of studies have discovered the association between Sox genes and tumorigenesis in various kinds of malignancies, including glioma, breast cancer, non-small cell lung cancer, gastric cancer, esophageal cancer, and colon rectal cancer. ${ }^{13-18}$ The most thoroughly studied member in this family is Sox2. Luo et al found that Sox2 was highly expressed in NPC compared with the nontumorous tissues. Furthermore, Sox2 correlated significantly with several clinical-pathological factors and epithelial-mesenchymal transition-associated indicators (E-cadherin/N-cadherin and Snail). ${ }^{19}$

With regard to Sox10, it was found to exist in various tissues, including heart, lungs, brain, adrenal glands, bladder, pancreas, salivary glands, colon, prostate, and testis in adulthood. The existence of Sox10 in visceral tissues was believed to be attribute to the presence of glial components in the peripheral nervous system in these tissues. ${ }^{20}$ Although many studies have discovered the role of Sox 10 mainly in melanoma, in salivary gland tumors, and brain tumors, like anaplastic oligodendroglioma, studies focusing on Sox 10 in NPC are limited..$^{8,21}$

Our study discovered that Sox 10 was highly expressed in NPC tissue, with staining found in both the nucleus and the cytoplasm. This is similar to the result of Sox10 staining in salivary adenoid cystic and breast basal-like carcinomas. ${ }^{22}$

We discovered that high expression of Sox 10 was correlated with advanced tumor stages and advanced $\mathrm{N}$ stage in NPC, which suggests that high expression of Sox 10 correlates with locally invasive features. Many studies showed the involvement of Sox10 in tumor aggressiveness. In melanoma, Hoek et al described that high levels of Sox10 have been correlated with a proliferative gene expression signature and low levels are associated with the gene expression profile of a highly invasive phenotype. ${ }^{23,24}$ Hoang et al' ${ }^{25}$ research retrospectively studied 428 cases of invasive ductal carcinoma of the breast. A significant association between Sox 10 expression and high-grade, estrogen receptor/progesterone receptor-negative, triple-negative, or basal-like invasive ductal carcinoma was discovered. These results may be explained by the mechanism in which Sox 10 works. One of the genes that is crucial in regulating the growth, differentiation, and survival of cells of the melanocytic lineage is microphthalmiaassociated transcription factor (MITF) ${ }^{26}$ One way that Sox 10 works is to act on MITF upstream, activate MITF expression, as well as synergize with MITF to activate downstream targets. ${ }^{27}$ Cronin et al's ${ }^{28}$ study discovered previously unknown mutations in MITF and Sox10. These mutations added up 
Table 2 Univariate and multivariate statistical analyses for various prognostic parameters in NPC patients

\begin{tabular}{|c|c|c|c|c|c|c|}
\hline \multirow[t]{2}{*}{ Characteristics } & \multirow[t]{2}{*}{$\mathbf{N}$} & \multicolumn{2}{|c|}{ Univariate analysis } & \multicolumn{3}{|c|}{ Multivariate analysis } \\
\hline & & $P$-value & $\begin{array}{l}\text { Regression } \\
\text { coefficient (SE) }\end{array}$ & $P$-value & Relative risk & $95 \% \mathrm{Cl}$ \\
\hline Expression of Sox 10 & & $0.005^{*}$ & $0.901(0.323)$ & $0.011 *$ & 2.3 & $1.215-4.353$ \\
\hline Low expression & 54 & & & & & \\
\hline High expression & 51 & & & & & \\
\hline Age (years) & & $0.027^{*}$ & $0.697(0.315)$ & 0.068 & 1.784 & $0.958-3.322$ \\
\hline$\leq 50$ & 77 & & & & & \\
\hline$>50$ & 28 & & & & & \\
\hline Sex & & 0.113 & $-0.552(0.348)$ & & & \\
\hline Male & 71 & & & & & \\
\hline Female & 34 & & & & & \\
\hline Clinical stage & & 0.11 & $0.318(0.199)$ & & & \\
\hline I & 3 & & & & & \\
\hline ॥ & 31 & & & & & \\
\hline III & 51 & & & & & \\
\hline IV & 20 & & & & & \\
\hline T classification & & 0.095 & $0.262(0.157)$ & & & \\
\hline TI & II & & & & & \\
\hline $\mathrm{T} 2$ & 58 & & & & & \\
\hline T3 & 18 & & & & & \\
\hline $\mathrm{T} 4$ & 18 & & & & & \\
\hline $\mathrm{N}$ classification & & 0.425 & $0.161(0.202)$ & & & \\
\hline No & 17 & & & & & \\
\hline $\mathrm{NI}$ & 43 & & & & & \\
\hline N2 & 43 & & & & & \\
\hline N3 & 2 & & & & & \\
\hline N0-I vs N2-3 & & 0.78 & $0.048(0.301)$ & & & \\
\hline N0-I & 60 & & & & & \\
\hline N2-3 & 45 & & & & & \\
\hline
\end{tabular}

Note: *Represents statistical significance.

Abbreviations: SE, standard error; $\mathrm{Cl}$, confidence interval.

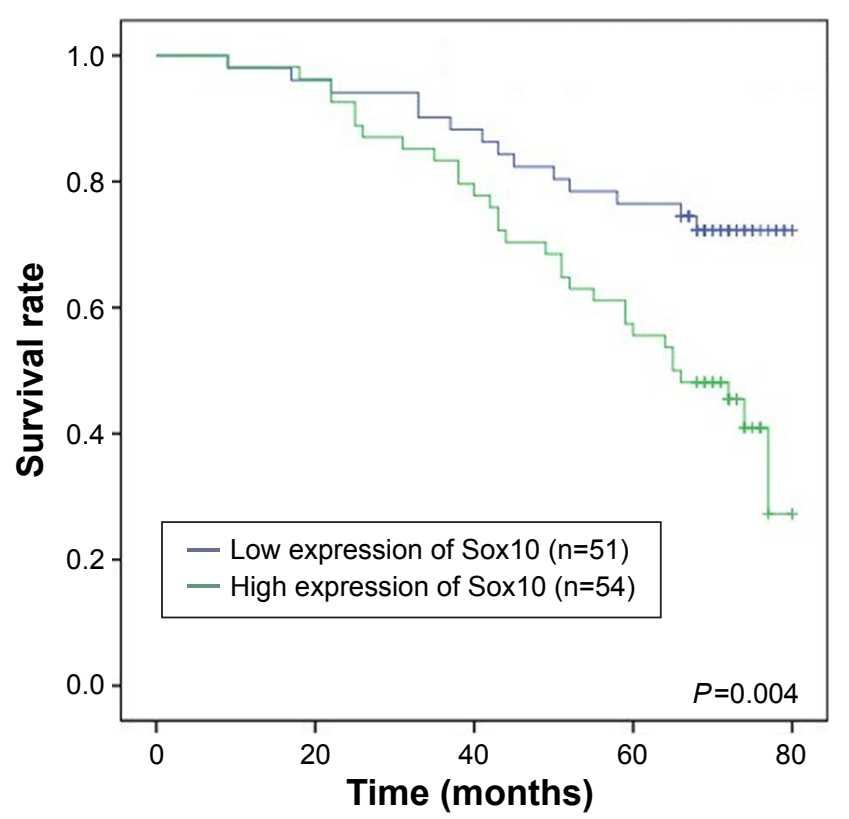

Figure 2 Survival analysis of 105 NPC patients by Kaplan-Meier method. Note: Survival curves of NPC patients with low expression versus high expression of Sox 10 ( $P=0.004$, log-rank test).

Abbreviation: NPC, nasopharyngeal carcinoma. to $>9 \%$ in primary melanoma tumors and $22 \%$ in metastatic melanoma tumors, which implied the involvement of MITF and Sox10 in melanoma.

Using Kaplan-Meier analysis, we discovered that high expression of Sox10 is associated with adverse overall survival and could be used as an independent prognostic marker. Our finding may be explained by the involvement of Sox10 in stem cell marker potential. Sox10 has been viewed as a stem cell marker and is found to be expressed extensively in melanoma. ${ }^{29}$ Studies also found that stable Sox10 knockdown in human melanoma cells arrested cell growth, altered cellular morphology and cell cycle, and induced senescence. ${ }^{30}$ Apart from in melanoma, ${ }^{31}$ Sox 10 has also shown its stem cell marker potential in breast cancer. Sox 10 was specifically expressed in mammary cells exhibiting the highest levels of stem/progenitor activity. This included fetal and adult mammary cells in vivo and mammary organoids in vitro. Sox10 was functionally relevant, as its deletion reduced stem/progenitor competence, whereas its overexpression increased stem/progenitor activity. ${ }^{32}$ 
Also, as a key downstream effector of Sox 9 in the mammary stem cell induction, Sox 10 promotes the tumorigenic and metastasis-seeding abilities of human breast cancer cells and is associated with poor patient survival. ${ }^{31}$ Tumor stem cells can lead to tumor cell proliferation and resistance to treatment. The mechanism on which Sox10 works on NPC remains to be explored by more work.

However, there are some limitations in our study. First, considering the favorable prognosis of patients diagnosed with NPC, we selected patients from 2004 to 2005 to better evaluate the prognosis. This resulted in incomplete data and inability to analyze progression-free survival because of lack of information in the Province Public Security Bureau and many people changed their phone numbers. Also, no patients included received concurrent chemoradiotherapy since it was not the standard treatment. From another viewpoint, this may better review the relationship between Sox10 expression and radiosensitivity. Second, the number of patients selected was 105. The results would be more convincing if more patients were included.

Next, we are planning to further validate the relationship between Sox10, radiosensitivity, and prognosis in NPC by downregulating (through knockdown) or upregulating Sox 10 expression in NPC cell lines.

\section{Conclusion}

In summary, we found that Sox10 was highly expressed in NPC and was associated with tumor aggressiveness and prognosis. It may serve as a potential biomarker for NPC patients. Finally, Sox10 may hopefully become a novel therapeutic target for NPC patients.

\section{Acknowledgments}

This work was partially supported by the National Key Clinical Specialty, Oncology Department (National Health and Family Planning Commission of the PRC 2013/544); the National Natural Science Foundation of China (Nos 81472802, 81201982, 81572500); the Specialized Research Fund for the Doctoral Program of Higher Education (No 20120171120110); and the Research Project of Health and Family Planning Commission of Hunan Province (No B2014-112).

\section{Disclosure}

The authors report no conflicts of interest in this work.

\section{References}

1. Torre LA, Bray F, Siegel RL, Ferlay J, Lortet-Tieulent J, Jemal A. Global cancer statistics, 2012. CA Cancer J Clin. 2015;65(2):87-108.
2. Aga M, Bentz GL, Raffa S, et al. Exosomal HIF1alpha supports invasive potential of nasopharyngeal carcinoma-associated LMP1-positive exosomes. Oncogene. 2014;33(37):4613-4622.

3. Lin DC, Meng X, Hazawa M, et al. The genomic landscape of nasopharyngeal carcinoma. Nat Genet. 2014;46(8):866-871.

4. Chan KC, Chan LS, Ip JC, et al. Therapeutic targeting of CBP/betacatenin signaling reduces cancer stem-like population and synergistically suppresses growth of EBV-positive nasopharyngeal carcinoma cells with cisplatin. Sci Rep. 2015;5:9979.

5. Gubbay J, Collignon J, Koopman P, et al. A gene mapping to the sexdetermining region of the mouse $\mathrm{Y}$ chromosome is a member of a novel family of embryonically expressed genes. Nature. 1990;346(6281): 245-250.

6. Mollaaghababa R, Pavan WJ. The importance of having your SOX on: role of SOX10 in the development of neural crest-derived melanocytes and glia. Oncogene. 2003;22(20):3024-3034.

7. Ng J, Celebre A, Munoz DG, Keith JL, Karamchandani JR. Sox10 is superior to $\mathrm{S} 100$ in the diagnosis of meningioma. Appl Immunohistochem Mol Morphol. 2015;23(3):215-219.

8. de la Rocha AM, Sampron N, Alonso MM, Matheu A. Role of SOX family of transcription factors in central nervous system tumors. $\mathrm{Am}$ J Cancer Res. 2014;4(4):312-324.

9. Bakos RM, Maier T, Besch R, et al. Nestin and SOX9 and SOX10 transcription factors are coexpressed in melanoma. Exp Dermatol. 2010;19(8): e89-e94.

10. Hoek KS, Eichhoff OM, Schlegel NC, et al. In vivo switching of human melanoma cells between proliferative and invasive states. Cancer Res. 2008;68(3):650-656.

11. Hoek KS, Schlegel NC, Brafford P, et al. Metastatic potential of melanomas defined by specific gene expression profiles with no BRAF signature. Pigment Cell Res. 2006;19(4):290-302.

12. Castillo SD, Sanchez-Cespedes M. The SOX family of genes in cancer development: biological relevance and opportunities for therapy. Expert Opin Ther Targets. 2012;16(9):903-919.

13. Garraway LA, Sellers WR. Lineage dependency and lineagesurvival oncogenes in human cancer. Nat Rev Cancer. 2006;6(8): 593-602.

14. Ben-Porath I, Thomson MW, Carey VJ, et al. An embryonic stem cell-like gene expression signature in poorly differentiated aggressive human tumors. Nat Genet. 2008;40(5):499-507.

15. Lengerke C, Fehm T, Kurth R, et al. Expression of the embryonic stem cell marker SOX2 in early-stage breast carcinoma. BMC Cancer. 2011;11:42.

16. Jeon HM, Sohn YW, Oh SY, et al. ID4 imparts chemoresistance and cancer stemness to glioma cells by derepressing miR- $9 *$-mediated suppression of SOX2. Cancer Res. 2011;71(9):3410-3421.

17. Sashikawa KM, Mutoh H, Sugano K. SOX9 is expressed in normal stomach, intestinal metaplasia, and gastric carcinoma in humans. J Gastroenterol. 2011;46(11):1292-1299.

18. Andersen CL, Christensen LL, Thorsen K, et al. Dysregulation of the transcription factors SOX4, CBFB and SMARCC1 correlates with outcome of colorectal cancer. Br J Cancer. 2009;100(3):511-523.

19. Luo W, Li S, Peng B, Ye Y, Deng X, Yao K. Embryonic stem cells markers SOX2, OCT4 and Nanog expression and their correlations with epithelial-mesenchymal transition in nasopharyngeal carcinoma. PLoS One. 2013;8(2):e56324.

20. Khong HT, Rosenberg SA. The Waardenburg syndrome type 4 gene, SOX10, is a novel tumor-associated antigen identified in a patient with a dramatic response to immunotherapy. Cancer Res. 2002;62(11): 3020-3023.

21. Ohtomo R, Mori T, Shibata S, et al. SOX10 is a novel marker of acinus and intercalated duct differentiation in salivary gland tumors: a clue to the histogenesis for tumor diagnosis. Mod Pathol. 2013;26(8): 1041-1050.

22. Ivanov SV, Panaccione A, Nonaka D, et al. Diagnostic SOX10 gene signatures in salivary adenoid cystic and breast basal-like carcinomas. Br J Cancer. 2013;109(2):444-451. 
23. Hoek KS, Eichhoff OM, Schlegel NC, et al. In vivo switching of human melanoma cells between proliferative and invasive states. Cancer Res. 2008;68(3):650-656

24. Hoek KS, Schlegel NC, Brafford P, et al. Metastatic potential of melanomas defined by specific gene expression profiles with no BRAF signature. Pigment Cell Res. 2006;19(4):290-302.

25. Hoang LL, Wang J, Tacha D, et al. Expression of SOX10 in invasive ductal carcinoma of the breast. Thirty-Seventh Annual CTRC-AACR San Antonio Breast Cancer Symposium[C]; December 9-13, 2014; San Antonio, TX.

26. Hou L, Pavan WJ. Transcriptional and signaling regulation in neural crest stem cell-derived melanocyte development: do all roads lead to MITF? Cell Res. 2008;18(12):1163-1176.

27. Ludwig A, Rehberg S, Wegner M. Melanocyte-specific expression of dopachrome tautomerase is dependent on synergistic gene activation by the Sox10 and MITF transcription factors. FEBS Lett. 2004; $556(1-3): 236-244$.
28. Cronin JC, Wunderlich J, Loftus SK, et al. Frequent mutations in the MITF pathway in melanoma. Pigment Cell Melanoma Res. 2009;22(4): 435-444.

29. Mohamed A, Gonzalez RS, Lawson D, Wang J, Cohen C. Tumor stem cells (CD271, c-kit, SOX10) in melanomas: prognostic and outcome implications. Appl Immunohistochem Mol Morphol. 2014;22(2): $142-145$.

30. Cronin JC, Watkins-Chow DE, Incao A, et al. SOX10 ablation arrests cell cycle, induces senescence, and suppresses melanomagenesis. Cancer Res. 2013;73(18):5709-5718.

31. Guo W, Keckesova Z, Donaher JL, et al. Slug and Sox9 cooperatively determine the mammary stem cell state. Cell. 2012;148(5) 1015-1028.

32. Dravis C, Spike BT, Harrell JC, et al. Sox10 regulates stem/progenitor and mesenchymal cell states in mammary epithelial cells. Cell Rep. 2015;12(12):2035-2048.

\section{Publish your work in this journal}

OncoTargets and Therapy is an international, peer-reviewed, open access journal focusing on the pathological basis of all cancers, potential targets for therapy and treatment protocols employed to improve the management of cancer patients. The journal also focuses on the impact of management programs and new therapeutic agents and protocols on

\section{Dovepress}

patient perspectives such as quality of life, adherence and satisfaction. The manuscript management system is completely online and includes a very quick and fair peer-review system, which is all easy to use. Visit http://www.dovepress.com/testimonials.php to read real quotes from published authors. 